\title{
TERAPIA MIOFUNCIONAL OROFACIAL EM CRIANÇAS RESPIRADORAS ORAIS
}

\section{Myofunctional therapy in children with oral breathing}

\author{
Júlia Gallo ${ }^{(1)}$, Alcione Ramos Campiotto ${ }^{(2)}$
}

\begin{abstract}
RESUMO
Objetivo: descrever a evolução de crianças respiradoras orais, submetidas à terapia miofuncional orofacial com ênfase no trabalho de fortalecimento da musculatura dos órgãos fonoarticulatórios e treino da respiração nasal. Métodos: participaram da pesquisa seis crianças, com idades entre 5 e 11 anos, que haviam realizado triagem no Setor de Terapia Fonoaudiológica da Irmandade Santa Casa de Misericórdia de São Paulo, durante o ano de 2007, que apresentavam respiração oral ou oronasal e sintomas e sinais característicos desta alteração, e não haviam realizado tratamento fonoaudiológico prévio. Os pacientes foram submetidos a 10 sessões de terapia miofuncional orofacial, enfocando o fortalecimento da musculatura dos órgãos fonoarticulatórios e treino da respiração nasal. Após as sessões, foi realizada reavaliação e a evolução dos pacientes foi comparada qualitativamente. Resultados: quatro $(66,66 \%)$ dos indivíduos apresentavam diagnóstico de obstrução nasal. Destes, três realizaram cirurgia prévia ao tratamento. Dois $(33,33 \%)$ apresentavam diagnóstico de rinite alérgica e estavam em tratamento na época das sessões. Todos apresentaram melhora satisfatória quanto aos aspectos trabalhados. Três (50\%) apresentavam deformidades dentofaciais e apresentaram evolução, porém limitada pela condição dentária. Todos os indivíduos apresentaram melhora no padrão de vedamento labial e possibilidade de respiração nasal. Conclusão: dez sessões de terapia miofuncional, com ênfase no fortalecimento da musculatura dos órgãos fonoarticulatórios e treino da respiração nasal foram suficientes para a obtenção da melhora dos pacientes. O pequeno número de sujeitos da amostra não nos permite generalizar a conclusão para outros. Sugere-se a realização de novos estudos, com maior população e diferentes faixas etárias.
\end{abstract}

DESCRITORES: Respiração Bucal; Terapia Miofuncional; Fonoterapia

\section{INTRODUÇÃO}

A função respiratória deve ocorrer por via nasal. O nariz, além de ser um condutor passivo pelo qual o ar é captado da atmosfera, é um órgão altamente especializado, capaz de realizar três importantes funções respiratórias: umidificação, aquecimento do ar inspirado e proteção das vias aéreas superiores ${ }^{1,2}$.

(1) Fonoaudióloga; Aperfeiçoanda em Motricidade Orofacial pela Irmandade Santa Casa de Misericórdia de São Paulo, ISCMSP, São Paulo, SP.

(2) Fonoaudióloga; Coordenadora do Curso de Aperfeiçoamento em Motricidade Oral em Fonoaudiologia da Irmandade da Santa Casa de Misericórdia de São Paulo, ISCMSP, São Paulo, SP; Mestre em Fonoaudiologia pela Pontifícia Universidade Católica de São Paulo.

Conflito de interesses: inexistente
Quando há algum impedimento para que a respiração nasal possa se realizar, tem-se a respiração oral. A respiração oral pode ser viciosa, ou seja, o indivíduo respira pela boca apesar de apresentar capacidade anatomofisiológica para respirar pelo nariz, ou orgânica, quando existem alterações orgânicas obstruindo a passagem de $\mathrm{ar}^{3}$. Independente da etiologia, a respiração pode ser afetada causando múltiplas alterações, com maior ou menor grau, em função do tempo de evolução do processo obstrutivo ${ }^{4}$.

A respiração de modo nasal possibilita o crescimento e desenvolvimento facial de maneira adequada, por meio da ação correta da musculatura. Nos casos de respiração oral, esta estimulação pode ocorrer de modo inadequado, influenciando negativamente o crescimento e o desenvolvimento do esqueleto craniofacial, principalmente nos aspectos de forma do maxilar, da mandíbula e da altura facial ${ }^{5-8}$. 
O diagnóstico, bem como o tratamento da respiração oral, devem ser realizados o mais precoce possível por uma equipe multidisciplinar, para que seja possível minimizar suas consequências ${ }^{\text {9-12. }}$.

A terapia miofuncional orofacial é considerada um método de tratamento que pode aumentar a força muscular, podendo devolver a estabilidade morfo-funcional às estruturas orofaciais. A terapia pode provocar mudanças nos padrões funcionais, e assim prevenir desvios no desenvolvimento craniofacial, pois promove nova postura de estruturas em repouso e durante a realização das funções do sistema estomatognático ${ }^{6,13}$.

Considerando as informações acima descritas, o objetivo deste estudo foi descrever a evolução de crianças de cinco a 11 anos, respiradoras orais, submetidas à terapia miofuncional orofacial com ênfase no trabalho de fortalecimento da musculatura dos órgãos fonoarticulatórios e no treino da respiração nasal.

\section{MÉTODOS}

A pesquisa foi realizada no Ambulatório de Motricidade Orofacial do Setor de Terapia Fonoaudiológica, Departamento de Otorrinolaringologia, da Irmandade Santa Casa de Misericórdia de São Paulo (ISCMSP), durante o ano de 2007.

Foi realizado um estudo prospectivo, para o qual foram selecionadas oito crianças, com idades entre cinco e 11 anos, que realizaram triagem no Setor de Terapia Fonoaudiológica - ISCMSP, no período de janeiro de 2005 a junho de 2007 e que estavam na fila de espera para o atendimento fonoaudiológico.

Critérios de inclusão: indivíduos com idades entre cinco e onze anos, que realizaram triagem no Setor de Terapia Fonoaudiológica - ISCMSP e apresentavam respiração oral ou oronasal e os sintomas e sinais característicos desta alteração, como dificuldade de vedamento labial, diminuição de força da musculatura dos órgãos fonoarticulatórios e não haviam realizado tratamento fonoaudiológico prévio.

Critérios de exclusão: não foram incluídos na amostra os indivíduos que apresentavam outras alterações além da respiração oral e seus sinais e sintomas característicos verificados na triagem.

Os sujeitos participantes da amostra e seus responsáveis receberam informações a respeito da pesquisa da qual participaram, e assinaram o Termo de Consentimento Livre e Esclarecido para autorização da sua participação no estudo.

Os participantes foram submetidos inicialmente a uma avaliação mais detalhada dos órgãos fonoarticulatórios, da capacidade de vedamento labial e avaliação da função nasal. Esta avaliação é composta por uma breve anamnese que continha os dados de identificação dos sujeitos e detaIhamento sobre a queixa que os levou à triagem. A segunda parte é composta por uma análise clínica, através de inspeção oral. Foi solicitado que os participantes se posicionassem sentados em cadeira comum, com os pés apoiados no chão, coluna ereta e cabeça em posição ortostática. Foram observados pela fonoaudióloga os aspectos referentes às estruturas do sistema sensório motor oral (tipo facial, posição de lábios e língua no repouso, características dos lábios superior e inferior, frênulo labial e lingual, tônus e mobilidade de lábios e língua, oclusão e funções orais como mastigação, deglutição, respiração e fala). A função nasal foi medida por meio da utilização do Espelho de Glatzel. Esta avaliação é parte do protocolo utilizado no Setor de Terapia Fonoaudiológica - ISCMSP.

Após a avaliação, deram início às dez sessões de terapia miofuncional orofacial, enfocando o trabalho de fortalecimento da musculatura dos órgãos fonoarticulatórios e treino da respiração nasal.

Os atendimentos foram realizados individualmente, uma vez por semana, em sessões de 30 minutos de duração cada. Na primeira sessão, e sempre que necessário, os pais receberam orientações quanto à realização dos exercícios em casa. Os exercícios eram selecionados de acordo com a idade de cada paciente. Para os pacientes com idades entre cinco e sete anos, os exercícios foram introduzidos por meio de atividade lúdica, para motivá-los a realizar os exercícios. Foram utilizados exercícios isométricos e isotônicos, semeIhantes aos utilizados na rotina do ambulatório de Terapia Fonoaudiológica, como por exemplo trabaIhar força de lábios e bochechas, alongamento de filtro de lábio superior, relaxamento da musculatura mentual, treino da respiração nasal, entre outros. Além disso, os pacientes foram orientados a realizar tratamento otorrinolaringológico para melhorar o aspecto da obstrução nasal. A cada sessão a terapeuta verificava a realização dos exercícios, corrigindo-os e alterando-os quando necessário. Após dez sessões, os indivíduos foram reavaliados utilizando-se a mesma avaliação inicial, com os mesmos critérios para possibilitar comparação dos resultados obtidos.

Este trabalho foi analisado e aprovado pelo Comitê de Ética em Pesquisa da Irmandade Santa Casa de Misericórdia de São Paulo (ISCMSP) sob o no 0270/07.

Os dados referentes à evolução dos pacientes pré e pós-terapia foram comparados e foi realizada uma análise descritiva e qualitativa dos resultados. 


\section{RESULTADOS}

Dos oito pacientes selecionados, dois desistiram logo após a realização da avaliação miofuncional, não iniciando as sessões de terapia. Devido às desistências, a amostra final foi composta por seis indivíduos.

Foram estudados seis pacientes, com idade entre cinco e 11 anos (média 8,16 anos), sendo cinco do sexo masculino e um do sexo feminino. Verificou-se que não houve predominância de uma determinada idade.

Dos seis indivíduos estudados, quatro apresentavam diagnóstico de obstrução nasal - hipertrofia de amídalas e adenoides (66,66\% dos indivíduos) e dois apresentavam diagnóstico de rinite alérgica e estavam em tratamento na época da realização das sessões de terapia (33,33\% dos indivíduos). Estes dados podem ser observados na Figura 1.

Dos quatro indivíduos com diagnóstico de obstrução nasal, três (75\%) realizaram cirurgia antes do início da terapia, e um (25\%) realizou a cirurgia após o término das sessões. Os pacientes que

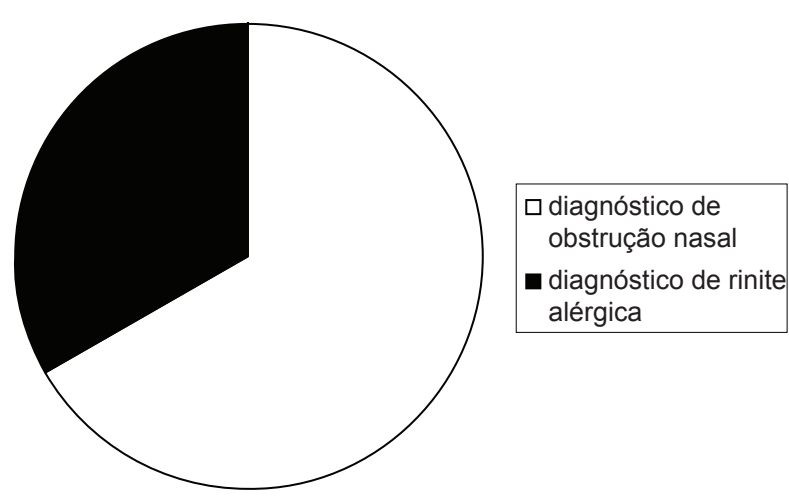

Figura 1 - Diagnóstico otorrinolaringológico dos pacientes estudados realizaram a cirurgia antes do início das sessões de terapia apresentaram evolução do padrão de vedamento labial e respiração nasal, porém cada um evoluiu de acordo com o limite permitido pela condição dentária apresentada. $O$ indivíduo que apresentava obstrução ainda na época da realização das sessões, evoluiu adequadamente e, após a cirurgia, apresentou melhora satisfatória que possibilitou respiração nasal na maior parte do tempo, além do aumento de força da musculatura dos órgãos fonoarticulatórios.

Observou-se deformidades dentofaciais em três dos indivíduos estudados (50\% dos indivíduos). As deformidades dentofaciais encontradas foram sobressaliência aumentada e mordida aberta anterior. Destes indivíduos, apenas um (33,33\%) estava realizando tratamento ortodôntico para correção da deformidade dentofacial.

Comparando as avaliações realizadas pré e pós-terapia, verificou-se que antes da terapia, $100 \%$ os indivíduos apresentavam pelo menos um padrão alterado, com relação ao vedamento labial, força da musculatura dos órgãos fonoarticulatórios, e respiração nasal. Ao final das dez sessões, apenas 33,33\% dos pacientes não apresentaram evolução satisfatória, o que pode ser justificado pela presença de deformidades dentofaciais e não realização de tratamento ortodôntico. $66,66 \%$ dos pacientes apresentaram melhora satisfatória do padrão de vedamento labial e possibilidade de respiração nasal na maior parte do tempo (Figura 2).

\section{DISCUSSÃO}

Devido aos critérios de exclusão selecionados para este estudo, houve dificuldade para compor a amostra, pois a maioria das crianças triadas no setor apresentava outras alterações e não puderam

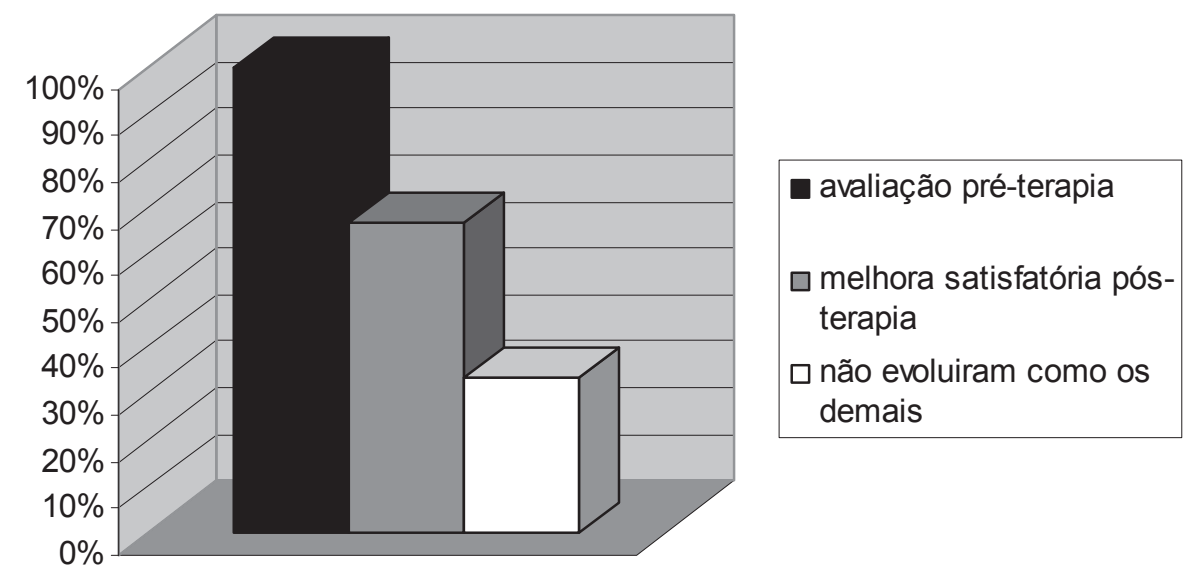

Figura 2 - Evolução dos pacientes após 10 sessões de terapia 
ser incluídas, uma vez que necessitariam de uma terapia fonoaudiológica mais completa, que deveria ser realizada em outro setor.

Todos os indivíduos mostraram-se participativos e motivados durante as sessões, realizando adequadamente os exercícios propostos pela terapeuta.

Neste estudo verificou-se que quatro $(66,66 \%)$ apresentaram obstrução nasal, que foi previamente corrigida com cirurgia. Sabe-se que as causas mais frequentes da respiração oral são obstruções nasais e/ou faríngeas ${ }^{14}$. A flacidez dos músculos faciais e mastigatórios também pode levar a boca a se abrir, podendo causar uma respiração oral funcional, neste caso não há qualquer obstrução ${ }^{9}$.

Todos os indivíduos participantes deste estudo apresentavam alteração de órgãos fonoarticulatórios e 50\% dos indivíduos apresentavam alterações dentofaciais. Os indivíduos respiradores orais podem apresentar vários sintomas característicos do quadro chamado de Síndrome do Respirador Oral, como: alterações craniofaciais e dentárias, alterações de órgãos fonoarticulatórios, entre outras ${ }^{15-17}$. Não é necessário que todos os sintomas estejam presentes para se diagnosticar um quadro de Síndrome do Respirador Oral ${ }^{18}$.

Dos indivíduos que apresentavam deformidades dentofaciais $(50 \%)$, apenas um estava realizando tratamento ortodôntico na ocasião das sessões de terapia. Verificou-se que o indivíduo em tratamento ortodôntico evoluiu de maneira mais satisfatória à terapia do que os indivíduos sem tratamento ortodôntico, apesar da deformidade dentofacial existente. A relação entre a respiração oral e as alterações no desenvolvimento facial já foi encontrada em outros estudos ${ }^{19,20}$.

Neste estudo, a terapia miofuncional orofacial realizada enfatizou o fortalecimento da musculatura dos órgãos fonoarticulatórios, além do treino da respiração nasal. Na literatura encontrou-se um estudo realizado para verificar a eficácia da terapia miofuncional nos casos de respiração oral em crianças. Os autores concluíram que, a terapia, enfatizando fortalecimento da musculatura dos órgãos fonoarticulatórios e realizada após eliminação de hábito de sucção, possibilitou a instalação da respiração nasal ${ }^{21}$.

Todos os indivíduos estudados apresentaram evolução quanto à força da musculatura dos órgãos fonoarticulatórios após as dez sessões de terapia. Autores realizaram um trabalho no qual utilizaram avaliação clínica e eletromiográfica dos músculos periorbiculares em crianças respiradoras bucais habituais, com o objetivo de verificar a influência da terapia miofuncional sobre estes músculos nas situações de repouso e vedamento labial. Os resultados mostraram que a terapia miofuncional pode melhorar a morfologia e as funções dos músculos em pacientes respiradores orais sem obstrução nasal ${ }^{22-24}$. Em um estudo realizado sobre comportamento dos músculos orbiculares da boca em crianças respiradoras orais pré e pós-mioterapia e concluíram que houve modificação na atividade dos músculos orbiculares superior e inferior da boca quando comparados antes e depois do tratamento miofuncional, aproximando o nível de atividade das crianças com respiração oral, com o nível das crianças com respiração nasal ${ }^{25}$.

Foi possível verificar que dez sessões de terapia miofuncional orofacial, com ênfase no fortalecimento da musculatura dos órgãos fonoarticulatórios, além do treino da respiração nasal, foram suficientes para a obtenção da melhora dos pacientes. Este achado está de acordo com um estudo realizado que verificou a evolução dos pacientes estudados após oito sessões de terapia miofuncional orofacial ${ }^{22}$, e também está de acordo com outro trabalho que verificou melhora no padrão de respiração diurna e postura labial em sessões semanais durante nove semanas, com outras subsequentes mensais para acompanhamento ${ }^{26}$.

$\mathrm{Na}$ literatura verificou-se que sintomas como baba noturna, ronco e alergia estão diretamente relacionados ao tipo respiratório, prevalecendo em sua maioria no grupo dos respiradores orais, apesar também de encontrá-los nos respiradores nasais ${ }^{27}$. O impacto da asma, rinite alérgica e respiração oral afetam diretamente a qualidade de vida do indivíduo não só pela alteração respiratória, mas, também pelos prejuízos comportamentais, funcionais e físicos que ocasionam. O controle dessas morbidades é um tema usual na literatura ${ }^{15,27,28}$.

\section{CONCLUSÃO}

Foi possível verificar melhora em todos os indivíduos quanto ao vedamento labial, aumento de força da musculatura dos órgãos fonoarticulatórios, além de maior possibilidade de respiração nasal após 10 sessões de terapia. O pequeno número de sujeitos dessa amostra, no entanto, não permite estabelecer que tais conclusões possam ser generalizáveis para outros grupos em diferentes faixas etárias. Sendo assim, sugere-se a realização de novos estudos, com maior população e de diferentes faixas etárias.

\section{AGRADECIMENTOS}

Às alunas e monitoras do Curso de Aperfeiçoamento em Motricidade Orofacial pela colaboração para a realização deste trabalho. 


\begin{abstract}
Purpose: to describe the evolution of children with oral breathing, submitted to myofuncional therapy with emphasis on strengthening the muscles of the phonoarticulatoty organs and training of nasal breathing. Methods: six children, 5 and 11-year old, who had performed screening at the Division of Phonoaudiological Therapy at Irmandade Santa Casa de Misericordia de São Paulo, along the year 2007, who demonstrated oral or oronasal breathing and signals and symptoms that are characteristic to this alteration, and had not been submitted to previous phonoaudiological treatment. The patients underwent 10 sessions of miofuntional therapy, focused on strengthening the muscles of the phonoarticulatory organs and training of nasal breathing. After the sessions, a reassessment was carried out and the evolution of the patients was compared qualitatively. Results: four $(66.66 \%)$ of the individuals were diagnosed with nasal obstruction. Three had been submitted to surgery previous to the phonoaudiological treatment. Two (33.33\%) had a diagnosis of allergic rhinitis and were undergoing a treatment concomitantly to the sessions. All showed satisfactory improvement as for the worked issues. Three $(50 \%)$ of the individuals had dentofacial deformities and made progress, though limited by the dental condition. All individuals showed improvement in the possibility of mouth closure and nasal breathing. Conclusion: ten sessions of miofuntional therapy, with emphasis on strengthening the muscles of the phonoarticulatory organs and training of nasal breathing were sufficient to obtain the improvement of the patients. The small number of the samples does not allow us to generalize the conclusion for others. We suggest to make further studies with larger populations and different age groups.
\end{abstract}

KEYWORDS: Oral Breathing; Myofuncional Therapy; Speech Therapy

\section{REFERÊNCIAS}

1. Marchesan IQ. Motricidade Oral: visão clínica do trabalho fonoaudiológico integrado com outras especialidades. São Paulo: Pancast; 1993. 781p.

2. Motonaga SM, Berti LC, Anselmo-Lima WT. Respiração bucal: causas e alterações no sistema estomatognático. Rev Bras Otorrinolaringol. 2000; 66(4):373-9.

3. Jabur LB. Avaliação fonoaudiológica. In: Ferreira FV, organizador. Ortodontia: diagnóstico e planejamento clínico. São Paulo: Artes Médicas; 1998. p. 283-309.

4. Novaes MSP, Vigorito JW. Respiração bucal: aspectos gerais e principais metodologias empregadas para avaliação. Ortodontia. 1993; 26(3):43-52.

5. Mitre El. Otorrinolaringologia e fonoaudiologia. São Paulo: Pulso; 2003. 138p.

6. Lessa FCR, Enoki C, Feres MFN, Valera FCP, Lima WTA, Matsumoto MAN. Influência do padrão respiratório na morfologia craniofacial. Rev Bras Otorrinolaringol. 2005; 71(2):156-60.

7. Coelho MF, Terra VHTC. Implicações clínicas em pacientes respiradores bucais. Rev Bras Patol Oral. 2004 jan/mar; 3(1):17-9.

8. Ribeiro EC, Marchiori SC, Silva AM. Electromyographics muscle EMG activity in mouth and nasal breathing children. Cranio. 2004; 22(2):145-50.

9. Junqueira $P$. Respiração oral: considerações fonoaudiológicas. In: Junqueira P, Dauden AT. Aspectos atuais em terapia fonoaudiológica II. São Paulo: Pancast; 2002. p. 9-16.

10. Marchesan IQ. A equipe de trabalho no respirador oral. In: Krakauer LH, Di Francesco RC, Marchesan IQ, organizador. Conhecimentos essenciais para entender bem a respiração oral. São José dos Campos: Pulso; 2003. p.163-7.

11. Amaral EC, Bacha SMC, Ghersel ELA, Rodrigues PMI. Inter-relação entre a odontologia e a fonoaudiologia na motricidade orofacial. Rev CEFAC. 2006; 8(3):328-36.

12. Degan VV, Puppin-Rontani RM. Remoção de hábitos e terapia miofuncional: restabelecimento da deglutição e repouso lingual. Pró-Fono. 2005; 17(3):375-82.

13. Degan VV: Hábitos de sucção e distúrbios miofuncionais orofaciais. In: Degan VV, Boni RC. Hábitos de sucção de chupeta e mamadeira. São José dos Campos: Pulso; 2004. p. 27-8.

14. Abreu RR, Rocha RL, Lamounier JA, Guerra AFM. Etiologia, manifestações clínicas e alterações presentes nas crianças respiradoras orais. J Pediatr. 2008; 84(6):529-35. 
15. Cattoni DM, Fernandes FD, Di Francesco RC, Latorre MRDO. Características do sistema estomatognático de crianças respiradoras orais: enfoque antroposcópico. Pró-Fono. 2007; 19(4):347-51.

16. Menezes VA, Leal RB, Pessoa RS, Pontes RMES. Prevalência e fatores associados à respiração oral em escolares participantes do projeto Santo Amaro-Recife, 2005. Rev Bras Otorrinolaringol. 2006; 72(3):394-9.

17. Prates NS, Magnani MBBA, Valdrighi HC. Respiração bucal e problemas ortodônticos: relação causa-efeito. Rev Paul Odontol. 1997; 19(4):14-9.

18. Rodrigues HOSN, Faria SR, Paula FSG, Motta AR. Ocorrência de respiração oral e alterações miofuncionais orofaciais em sujeitos em tratamento ortodôntico. Rev CEFAC. 2005; 7(3):356-62.

19. Amaral CSF, Martins ER, Rios JBM. A respiração bucal e o desenvolvimento do complexo dentofacial. Rev Bras Alergia Imunopatol. 2002; 25(4):131-5.

20. Andrade FV, Andrade DV, Araújo AS, Ribeiro ACC, Deccax LDG, Nemr K. Alterações estruturais de órgãos fonoarticulatórios e más oclusões dentárias em respiradores orais de 6 a 10 anos. Rev CEFAC. 2005; 7(3):318-25.

21. Bottero E, Ansanelli LSC, Motta AR. Ocorrência de respiração oral em pacientes com doenças periodontais. Rev CEFAC. 2005; 7(3):363-70.

22. Degan VV, Puppin-Rontani RM. Aumento da aeração nasal após remoção de hábito de sucção e terapia miofuncional. Rev CEFAC. 2007; 9(1):55-60. dx.doi.org/10.1590/S1516-18462007000100008
23. Schievano D, Rontani RMP, Berzin F. Influence of myofunctional therapy on the perioral muscles. Clinical and electromyographic evaluations. J Oral Rehabil. 1999; 26(7):564-9.

24. Degan VV, Puppin-Rontani RM. Terapia miofuncional e hábitos orais infantis. Rev CEFAC. 2004; 6(4):396-404.

25. Junqueira $P$, Parro FM, Toledo RM, Araújo RLT, Di Francesco RD, Rizzo MC: Conduta fonoaudiológica para pacientes com diagnóstico de rinite alérgica: relato de caso. Rev CEFAC. 2005; 7(3):336-9.

26. Silva AMT. Eletromiografia: avaliação dos músculos orbiculares da boca em crianças respiradoras bucais, pré e pós mioterapia. [tese]. São Paulo (SP): Universidade Federal de São Paulo; 2000.

27. Ríspoli CM, Bacha SM. Terapia miofuncional: intervenção breve. In: Marchesan IQ, Zorzi JL, Gaves IC.Tópicos em fonoaudiologia 1997/1998. vol 4. São Paulo: Lovise; 1998. p. 545-86.

28. Campanha SMA, Freire LMS, Fontes MJF. O impacto da asma, da rinite alérgica e da respiração oral na qualidade de vida de crianças e adolescentes. Rev CEFAC. 2008; 10(4):513-9. dx.doi.org/10.1590/S1516-18462008000400011 29. Lasmar LMLBF, Camargos PAM, Ordones AB, Gaspar GR, Campos EG, Ribeiro GA. Prevalência da rinite alérgica e seu impacto na utilização dos serviços de pronto-atendimento em um grupo de crianças e adolescentes com asma persistente moderada e grave. J Pediatr. 2007; 83(6):555-61.

RECEBIDO EM: 17/07/2008

ACEITO EM: 15/06/2009

Endereço para correspondência:

Júlia Gallo

Rua Duílio, 529 BI A ap. 42

São Paulo - SP

CEP: 05043-020

E-mail: ju_fonopauli@yahoo.com.br julinha_g@ hotmail.com 